\title{
INTENSE ELECTRON BEAM DISRUPTION DUE TO ION RELEASE FROM SURFACES
}

\author{
H. A. Davis, C. Vermare, T. P. Hughes, D. C. Moir, R. T. Olson and W. M. Wood \\ Los Alamos National Laboratory, Los Alamos, NM, USA
}

\begin{abstract}
A major concern for the DARHT second axis $(2 \mathrm{kA}$, $18.6 \mathrm{MeV}, 2000 \mathrm{~ns}$ ) is that ions or ionized neutrals released from solid surfaces (e.g., apertures, septums, dumps, and targets) by beam impact can be accelerated and trapped by the beam potential. This positive charge could disrupt the beam. To study this, experiments were performed on the DARHT first axis. The beam, focused to a range of diameters, is transmitted through thin foils made of various materials. The time-dependent beam radial profile is measured downstream of the target. For low current density, the downstream-beam profile is time invariant as expected. At higher current density, the downstream-beam radius changes during the pulse followed by transverse instability.
\end{abstract}

\section{INTRODUCTION}

The Dual-Axis Radiographic Hydrodynamics Testing (DARHT) facility will use two perpendicular electron Linear Induction Accelerators (LIA) to produce intense bremsstrahlung x-ray pulses for flash radiography of dense objects. The operational first axis produces a single electron pulse with $1.7 \mathrm{kA}$ current, $19.8 \mathrm{MeV}$ energy and $60 \mathrm{~ns}$ pulse duration. The second axis, under construction, will generate a $2.0 \mathrm{kA}, 19.8 \mathrm{MeV}, 2000 \mathrm{~ns}$ beam. A sequence of four shorter-duration pulses from this beam will be delivered to the x-ray converter by a fast kicker system. The second axis differs from previous LIA's by its much longer pulse duration, typically 10-20 times longer.

Ions created at a surface by beam impact are accelerated and trapped in the beam channel. These ions partially neutralize the beam space charge upsetting its equilibrium [1][2]. A relatively small ion charge density relative to the electron charge density (of order $1 / \gamma^{2}$ ) can upset the equilibrium for high $\gamma$ beams. On the second axis of DARHT, there are number of places where beam interception is expected. To study these effects, we have used the first axis DARHT beam as a probe. The beam is focused onto a thin foil (or mesh) to minimize the beam scattering. The mean current density can be varied over a wide range with an upstream focusing magnet. The timedependent behaviour of the beam radial profile is observed some distance downstream to assess the effects of ion release, from the foil or mesh, on the beam dynamics. The observations are compared with Particle- In-Cell (PIC) computer modelling to study ion emission at the foil (mesh).

\section{EXPERIMENTAL SET-UP AND PIC CODE}

\subsection{Beam line and diagnostics}

The DARHT first axis accelerator is an induction LIA composed of a $4 \mathrm{MV}$ injector (1.7kA, 80ns) and 64 induction cells. The set-up used for the experiment described here (cf. Figure 1) is located after the last induction cell where the beam energy is $19.8 \mathrm{MeV}$. This part of the beam line consists of two cryogenic pumps able to establish a vacuum better than $2 . \times 10^{-6}$ Torr, three Beam Position Monitors (BPM), a spinning wheels system that prevents target debris from damaging the accelerator, a magnetic solenoid, a foil chamber, a diagnostic section, and a beam stop. The beam-focusing solenoid, DT\#1, allows the mean beam current density at the foil to be adjusted from $0.5 \mathrm{~A} / \mathrm{mm}^{2}$ to $100 \mathrm{~A} / \mathrm{mm}^{2}$.

Figure 2 shows the Root Mean Square (RMS) beam radius at the foil location for different DT\#1 currents (measured with the Cherenkov diagnostic described below). The experimental results (symbols) are compared with beam envelope code predictions (lines). The beam current is constant between $\mathrm{t}=20 \mathrm{~ns}$ and $\mathrm{t}=80 \mathrm{~ns}$ as expected since the beam energy is constant to $1 \%$ over this time. The measurements and calculations show good agreement for $r>3 \mathrm{~mm}$. For smaller beam radius, the measured beam radius is a factor of two larger than the calculated radius. This discrepancy can be explained in part by the $\sim 1 \mathrm{~mm}$ spatial resolution of the diagnostic.

The foil is placed normal to the accelerator axis with the holder providing a symmetric return current connection. Seven foil materials were tested: graphite, aluminium, titanium, 304 stainless steel, molybdenum, tantalum, and gold. In addition, two mesh materials were tested: titanium and tungsten. The three-part detector located $660 \mathrm{~mm}$ downstream of the foil is composed of a thin $(10 \mu \mathrm{m})$ aluminized Kapton foil, a graphite disk ( $1 \mathrm{~mm}$ thick), and a $1 \mathrm{~mm}$ diameter quartz fiber placed vertically through the beam axis. This detector is tilted $43.6 \mathrm{deg}$ with respect to the accelerator axis to image the Optical Transition Radiation (OTR) produced at the vacuum-aluminium interface with a $500 \mathrm{~ms}$ exposure camera. This camera detects the time average beam distribution. A streak camera images the Cherenkov radiation from the fiber yielding the beam radial profile versus time. The three BPM's, placed before and after DT\#1 and between the foil and the detector, measure the 


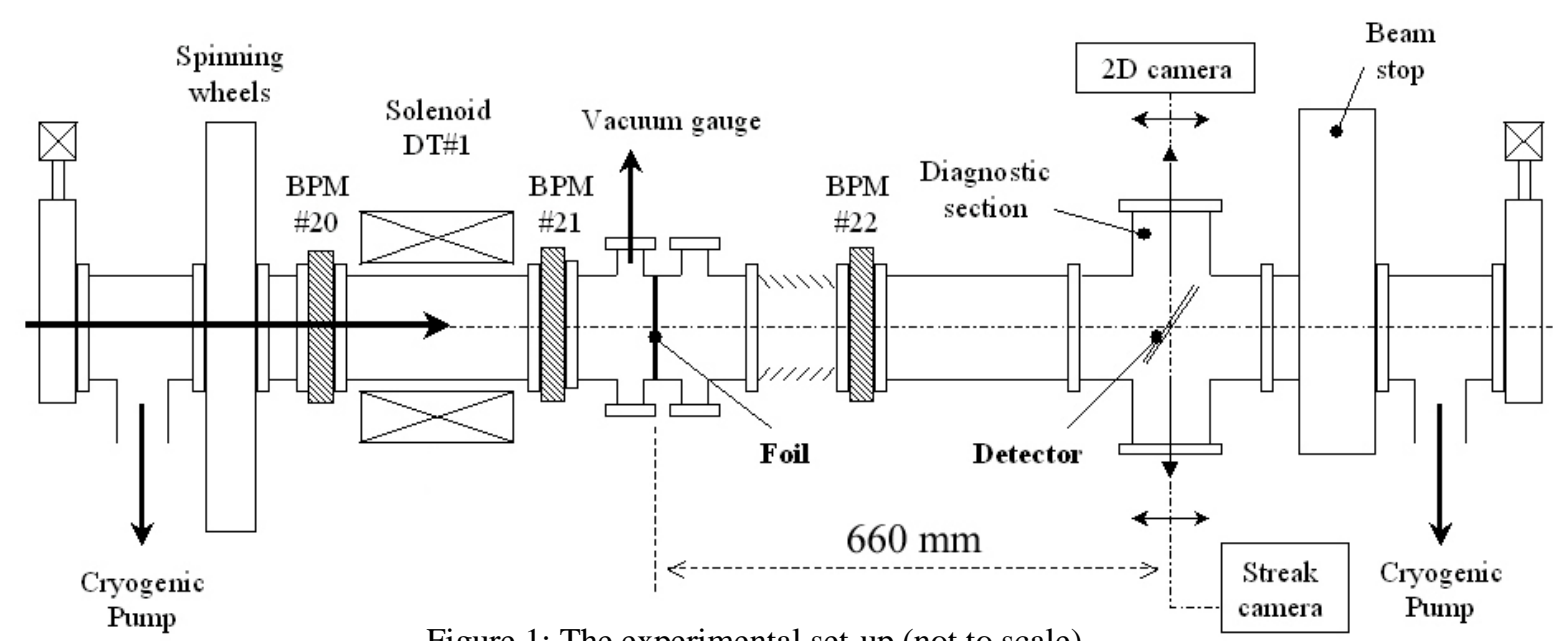

Figure 1: The experimental set-up (not to scale).

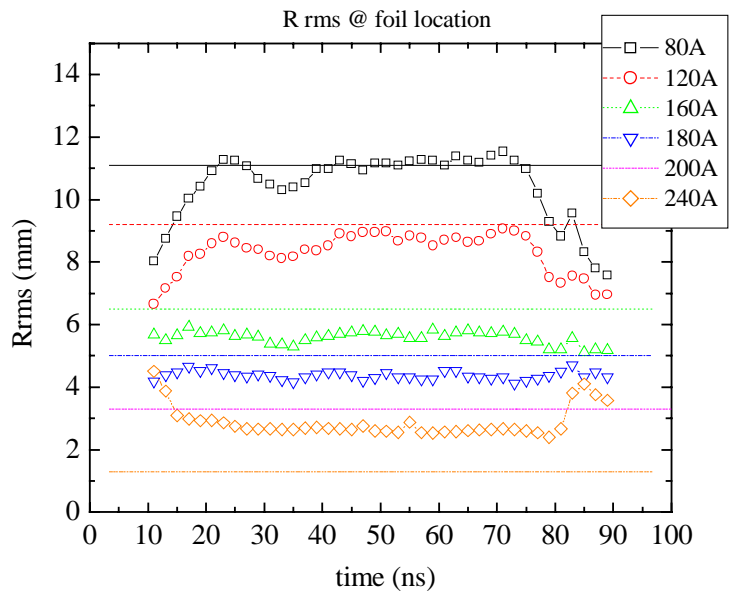

Figure 2: RMS beam radius versus time at the foil location for different DT\#1 solenoid currents.

total current and the beam centroid position versus time. A $94 \mathrm{GHz}$ microwave interferometer measures transient plasma density $3 \mathrm{~cm}$ from the foil surface. The microwave horn's transmission axis is parallel to the foil. Assuming the plasma is confined within a $1 \mathrm{~cm}$ radius of beam center, the interferometer is sensitive to average freeelectron densities greater than $10^{10} \mathrm{~cm}^{-3}$.

\subsection{Particle-In-Cell code description}

To model the beam-foil interaction, we used the parallelized 2-D/3-D particle-in-cell code Lsp [3]. The code has models for multiple scattering and energy-loss in materials, surface heating, particle emission from surfaces due to thermal and stimulated desorption, field emission, etc. An explicit electromagnetic charge-conserving algorithm was used. An electron beam with a Gaussian profile was injected upstream. The foil was modelled as a conducting plane with scattering and energy-loss corresponding to 7.6-thick- $\mu \mathrm{m}$ Al. Ions are allowed to emit at the space-charge-limited current density when the local foil temperature increase exceeds $300 \mathrm{~K}$. Calculations were for singly ionized $\mathrm{H}, \mathrm{C}$, and $\mathrm{Al}$ ions.

\section{RESULTS ANALYSIS}

\subsection{Time delay on the ions production}

Figure 3 displays the streak camera images recorded for different DT\#1 currents. For brevity only four foil materials are presented. Each frame (corresponding to a beam pulse) gives the vertical profile of the beam versus time. The spatial scale is indicated at the top right corner. Some horizontal lines are present due to imperfections in the fiber. On each frame, a vertical line corresponds to the beam profile at a given time. By assuming beam axial symmetry, the time dependence of light collected can be compared with the beam current. This comparison provides a check to insure that the beam profile measurement is not influenced by beam motion in the horizontal direction. For each material, there is a threshold value for the current density (given by the DT\#1 current setting) at the foil. For current densities less than or equal to this value, the beam profile is constant over the $60 \mathrm{~ns}$ beam current plateau. In this case, the beam profile at the detector depends on the foil focusing effect [4] and foil scattering. Above this current density threshold, the beam profile becomes time dependent part way through the pulse. The delay for onset of this phenomenon is dependent on the foil material (cf. arrows on Fig. 3).

Studies of anode plasma formation in diodes have produced strong evidence that a surface bombarded by an intense electron beam begins to emit ions after heating to $400 \pm 60^{\circ} \mathrm{C}$ [5]. Others have proposed that ions from the foil material are generated after the foil material melts [6]. In the former model, the foil temperature rise leads to thermal desorption of impurities absorbed on the surface that are subsequently ionized [7]. Ionization can be due to either beam impact or to a cascade process in the high electric field at the foil surface. The impurities are typically water and hydrocarbons. The observed delay corresponds to combination of the time needed to build a desorbed gas layer in front of the surface, the ionization 


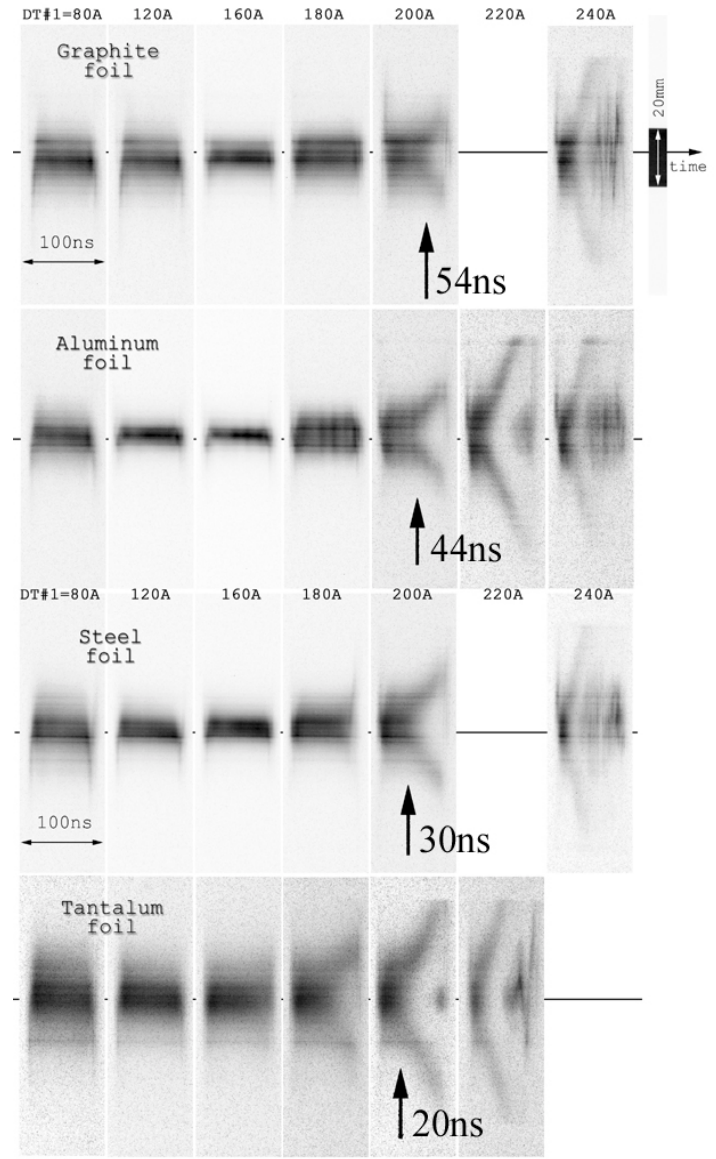

Figure 3: Streak camera images for different DT\#1 current setting and foil materials

time of the gas and the time for ions to move into the beam channel.

Table 1 shows the estimated time delays needed to heat the center of the foil to $400^{\circ} \mathrm{C}$ or to the melting point, for DT\#1 currents of $200 \mathrm{~A}$ and $240 \mathrm{~A}$. These estimations are based on the temperature rise of the central part of a Gaussian profile with the calculated RMS radius (cf. Section 2.1). We used the calculated radius because of the resolution limitations on the measured values for small radius. The delays needed to melt the foil are longer than

Table 1: Time delay (ns) to heat foils to $400^{\circ} \mathrm{C}$ or melting.

\begin{tabular}{|c|c|c|c|c|}
\hline $\begin{array}{c}\text { Material } \\
\text { (Melting temp. in } \\
\text { deg. }{ }^{\circ} \text { C) }\end{array}$ & \multicolumn{2}{|c|}{$\begin{array}{c}\text { DT\#1=200A } \\
\left(50 \mathrm{~A} / \mathrm{mm}^{2}\right)\end{array}$} & \multicolumn{2}{c|}{$\begin{array}{c}\text { DT\#1=240A } \\
\left(320 \mathrm{~A} / \mathrm{mm}^{2}\right)\end{array}$} \\
\cline { 2 - 5 } & $400^{\circ} \mathrm{C}$ & Melt & $400^{\circ} \mathrm{C}$ & Melt \\
\hline Graphite (3352) & 52 & $>200$ & 8 & 125 \\
\hline Aluminum (6959) & 44 & 80 & 7 & 12 \\
\hline Steel (1535) & 26 & 160 & 4 & 25 \\
\hline Tantalum (3020) & 8 & 70 & 1 & 11 \\
\hline
\end{tabular}

the observed delays and in some case longer than the beam pulse. The delays need to reach $400^{\circ} \mathrm{C}$ are comparable to the measured delays. This indicates that ionization and ion motion is rapid or that the $400^{\circ} \mathrm{C}$ temperature is somewhat higher than the actual temperature for desorption. After the expansion, the beam radius comes back to its original value. During this phase, a transverse oscillation of the beam centroid grows from the noise level $(\sim 1 \mathrm{~mm})$ to $10 \mathrm{~mm}$. Measurements with the interferometer showed that free-electron densities above the instrumental noise level of $10^{10} \mathrm{~cm}^{-3}$ were not observed prior to melting the foil.

\subsection{Comparison with PIC simulations - Nature of the ions}

The measured beam RMS radii versus time (DT\#1=200 A) are presented in the Figure 4. After expansion begins, the time dependence of the beam radius can be approximated with a linear fit (constant expansion velocity). Computer calculations show this velocity

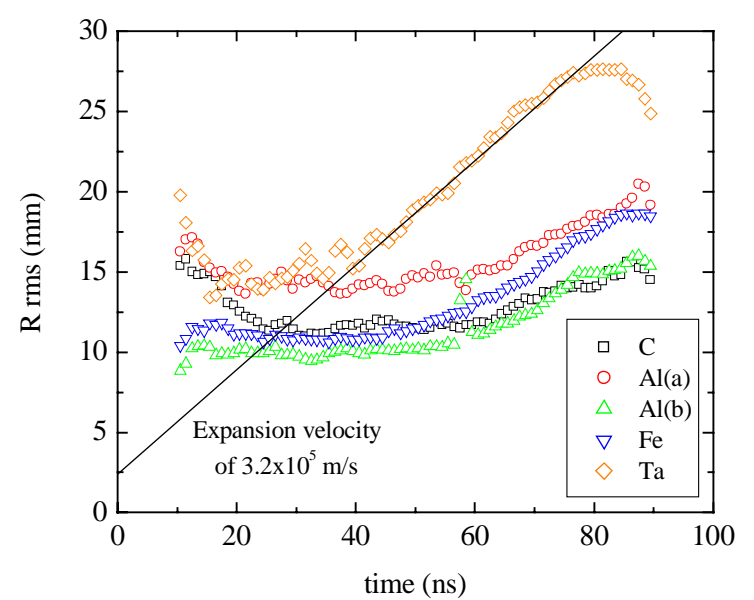

Figure 4: Beam RMS radius at the detector

depends on the mass of the ions emitted by the foil and the current density of the beam. For DT\#1=200 A, PIC simulations assuming space charge limited emission give: $8.10^{5} \mathrm{~m} / \mathrm{s}$ for $\mathrm{H}^{+}$ions, $4.10^{5} \mathrm{~m} / \mathrm{s}$ for $\mathrm{C}^{+}$ions and $2.710^{5} \mathrm{~m} / \mathrm{s}$ for $\mathrm{Al}^{+}$ions. Experimental results range from 2.6 to $3.210^{5} \mathrm{~m} / \mathrm{s}$ independent of the foil material. This comparison excludes very light ions (e.g., $\mathrm{H}^{+}, \mathrm{H}_{2}^{+}$) and suggests a ratio of charge state to atomic weight around 1/25. Many impurities molecules such as $\mathrm{C}_{2} \mathrm{H}_{\mathrm{m}}^{+}, \mathrm{H}_{2} \mathrm{O}^{+}$, $\mathrm{CO}^{+}, \mathrm{OH}^{+}$, etc fall into this range.

\section{REFERENCES}

[1] D. R Welch and T. P. Hughes, Laser and Particle Beams, 16, 285, (1998).

[2] C. Vermare et al, IEEE Transaction on Plasma Science, Vol. 27, \#06, p. 1566, (1999).

[3] T. P. Hughes, R. E. Clark, and S. S. Yu, Phys. Rev. ST Accel. Beams 2, 110401 (1999).

[4] R. F. Fernsler, R. F. Hubbard, and S. P. Slinker, J. Appl. Phys. 68, 12, (1990).

[5] T. W. L Sanford et al., J. Appl. Phys. 66, 10 (1989).

[6] T. J. Kwan et al., Phys. Of Plasmas, Vol. 7, \#05, p. 2215, (2000).

[7] M. E. Cuneo, IEEE Transaction on Dielectrics and Electrical Insulation, Vol. 6, \#04, p. 469, (1999). 